\title{
Correction to: When Transparency Clouds Rather Than Clarifies: A Closer Look at Transparency Bias Within ESG Scores
}

\author{
Ruben Feldman
}

\section{Correction to:}

\section{Chapter 13 in: K. Wendt (ed.),}

Theories of Change, Sustainable Finance, https://doi.org/10.1007/978-3-030-52275-9_13

The original version of the chapter was inadvertently published with an error. The author name has now been corrected to "Ruben Feldman". 